\title{
U-shaped association between plasma sphingosine-1-phosphate levels and mortality in patients with chronic systolic heart failure: a prospective cohort study
}

Yanbo Xue ${ }^{1 \dagger}$, Wei Jiang ${ }^{2 \dagger}$, Qiong Ma', Xiqiang Wang ${ }^{1}$, Pu Jia ${ }^{3}$, Qiang $\mathrm{Li}^{4}$, Shuping Chen ${ }^{1}$, Bingxue Song ${ }^{5}$, Ya Wang ${ }^{1}$, Jingwen Zhang ${ }^{6}$, Jing Liu', Guodong Yang ${ }^{1}$, Yuyao Lin' ${ }^{1}$, Jing Liu', Linyan Wei', Caijuan Dong , Haiquan $\mathrm{Li}^{1}$, Zhonglei Xie', Ling Bai ${ }^{1 *}$ and Aiqun $\mathrm{Ma}^{1{ }^{*}}$

\begin{abstract}
Background: The endogenous lipid molecule sphingosine-1-phosphate (S1P) has received attention in the cardiovascular field due to its significant cardioprotective effects, as revealed in animal studies. The purpose of our study was to identify the distribution characteristics of S1P in systolic heart failure patients and the prognostic value of S1P for long-term prognosis.

Methods: We recruited 210 chronic systolic heart failure patients from June 2014 to December 2015. Meanwhile 54 healthy people in the same area were selected as controls. Plasma S1P was measured by liquid chromatographytandem mass spectrometry. Patients were grouped according to the baseline S1P level quartiles, and restricted cubic spline plots described the association between S1P and all-cause death. Cox proportional hazard analysis was used to determine the relationship between category of S1P and all-cause death.
\end{abstract}

Results: Compared with the control group, the plasma S1P in chronic heart failure patients demonstrated a higher mean level $(1.269 \mu \mathrm{mol} / \mathrm{L}$ vs $1.122 \mu \mathrm{mol} / \mathrm{L}, P=0.006)$ and a larger standard deviation $(0.441 \mathrm{vs} 0.316, P=0.022)$. Based on multivariable Cox regression with restricted cubic spline analysis, a non-linear and U-shaped association between S1P levels and the risk of all-cause death was observed. After a follow-up period of $31.7 \pm 10.3$ months, the second quartile $(0.967-1.192 \mu \mathrm{m} / \mathrm{L})$ with largely normal S1P levels had the lowest all-cause mortality and either an increase (adjusted $\mathrm{HR}=2.368,95 \% \mathrm{Cl} 1.006-5.572, P=0.048$ ) or a decrease (adjusted $\mathrm{HR}=0.041,95 \% \mathrm{Cl} 0.002-0.808$, $P=0.036$ ) predicted a worse prognosis. The survival curves showed that patients in the lowest quartile and highest quartile were at a higher risk of death.

(Continued on next page)

\footnotetext{
*Correspondence: bailingb21@sina.com; aiqun.ma@xjtu.edu.cn; maaiqun1108@126.com

${ }^{\dagger}$ Yanbo Xue and Wei Jiang contributed equally to this work.

'Department of Cardiovascular Medicine, First Affiliated Hospital of Xi'an Jiaotong University; Shaanxi Key Laboratory of Molecular Cardiology; Key Laboratory of Environment and Genes Related to Diseases, Ministry of

Education, No. 277 West Yanta Road, Xi'an 710061, Shaanxi Province, People's Republic of China

Full list of author information is available at the end of the article
}

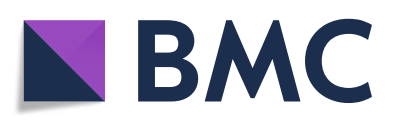

( ) The Author(s). 2020 Open Access This article is licensed under a Creative Commons Attribution 4.0 International License, which permits use, sharing, adaptation, distribution and reproduction in any medium or format, as long as you give appropriate credit to the original author(s) and the source, provide a link to the Creative Commons licence, and indicate if changes were made. The images or other third party material in this article are included in the article's Creative Commons licence, unless indicated otherwise in a credit line to the material. If material is not included in the article's Creative Commons licence and your intended use is not permitted by statutory regulation or exceeds the permitted use, you will need to obtain permission directly from the copyright holder. To view a copy of this licence, visit http://creativecommons.org/licenses/by/4.0/. The Creative Commons Public Domain Dedication waiver (http://creativecommons.org/publicdomain/zero/1.0/) applies to the data made available in this article, unless otherwise stated in a credit line to the data. 
(Continued from previous page)

Conclusions: Plasma S1P levels in systolic heart failure patients are related to the long-term all-cause mortality with a U-shaped correlation.

Trial registration: CHICTR, ChiCTR-ONC-14004463. Registered 20 March 2014.

Keywords: Heart failure, Sphingosine-1-phosphate, All-cause mortality, Prognosis

\section{Introduction}

Heart failure is the terminal state of a variety of cardiovascular diseases that affects the health of patients and increases the burden on society. Despite formal drug treatments, the 5-year mortality of heart failure patients remains high [1, 2]. Searches for new biomarkers with long-term prediction value and early identification of high-risk patients to reduce mortality are always important for guiding clinical treatments. Heart failure activates a variety of systemic compensatory stress responses, including stimulation of the sympathetic catecholamine system, the renin angiotensin aldosterone system and other systems. In recent years, a large number of studies have found that immune system activation and dysfunction are present during the occurrence and development of heart failure, and these immunity disorders always occur simultaneously with abnormalities of the neurohumoral endocrine system. Therefore, in-depth investigations of these pathophysiological changes call for appropriate molecules to connect the two systems together.

The circulating immunoregulatory factor sphingosine1-phosphate (S1P), an endogenous lipid molecule, is produced by the phosphorylation of sphingosine by sphingosine kinases. S1P is an intracellular secondary messenger, and it can also enter the circulation and bind to the G protein-coupled receptor family of sphingosine1-phosphate receptors $1-5$ to play an immunomodulatory role $[3,4]$. In recent years, studies have shown that S1P interacts with $\beta$-adrenergic receptors to play a role in simultaneously regulating immune system and sympathetic nervous system functions [5, 6]. Meanwhile, S1P has received attention due to its important role in cardiac ischaemia-reperfusion injury $[7,8]$ and ventricular remodelling inhibition $[9,10]$. It is interesting that previous in vitro and in vivo studies of S1P levels and its receptor targeting activities reveal a common feature that whether the S1P level is elevated or depressed in the experimental group, the heart-protecting effect is always achieved through adjusting the S1P/S1PR axis function to the level of the normal control group. These results suggest that keeping S1P levels in the normal range may have protective effects on the heart $[11,12]$.

Until now, it has been unclear whether there are abnormal S1P levels in clinical patients with chronic heart failure (CHF), what its normal level is, what its clinical significance is, and whether it is related to poor prognosis. The purpose of our study was to identify the distribution characteristics of S1P levels in the blood circulation of outpatients with stable systolic heart failure and its prognostic value for long-term prognosis.

\section{Methods}

\section{Patient selection}

We recruited systolic heart failure patients treated in Jingyang Hospital and Xunyi Hospital, both located in Shaanxi Province, and who were diagnosed according to the "2014 China Heart Failure Diagnosis and Treatment Guideline" and "2012 ESC Guidelines for the Diagnosis and Treatment of Acute and Chronic Heart Failure" [13] before and treated in the outpatient clinic from June 2014 to December 2015. The inclusion criteria were as follows: 1.18 to 80 years of age; 2. Echocardiography showing left ventricular systolic dysfunction $(\mathrm{EF}<50 \%)$; 3. Not hospitalized in the past month; and 4. Standardized drug treatment regimens. Patients were excluded if they met any of the following criteria: 1 . Systolic blood pressure $<90 \mathrm{mmHg}$ or haemodynamic instability; 2 . Second-degree type II AV block or severer; 3. HR < 55 beats/min in conscious resting state; 4 . Significant abnormal liver or kidney function; 5. Malignancy; 6 . Pregnant or lactating women; and 7. Dementia or mental disorder. The study design and population are detailed in Fig. 1.

All participants signed written informed consent forms. The study conformed to the principles outlined in the Declaration of Helsinki and was approved by relevant ethical bodies. Registration site: http://www.chictr. org.cn. Registration number: ChiCTR-ONC-14004463.

\section{Procedural management}

A group of senior professors from The First Affiliated Hospital of Xi'an Jiaotong University formed a coaching team to conduct unified and standardized training for the clinicians of the two hospitals through centralized training and prescription instruction before patient enrolment, according to the guidelines [13] to ensure consistent treatment management principles to reduce deviations in patient treatment regimens arising from different medical units and physicians. The biomarker data generated in this prospective study were not used for treatment decisions. 
274 patients treated in the outpatient clinic from June 2014 to December 2015

\section{7 patients satisfied the inclusion criteria}

\section{Inclusion criteria}

1.18 to 80 years of age.2. Echocardiography showing left ventricular systolic dysfunction $(\mathrm{EF}<50 \%)$. 3. Not hospitalized in the past month. 4. Standardized drug treatment regimens.

\section{Exclusion criteria}

1. Systolic blood pressure $<90 \mathrm{mmHg}$ or haemodynamic instability; 2. Second-degree type II AV block or severer; 3. $\mathrm{HR}<55$ beats/min in conscious resting state; 4 . Significant abnormal liver or kidney function; 5. Malignancy; 6.Pregnant or lactating women; 7. Dementia or mental disorder.

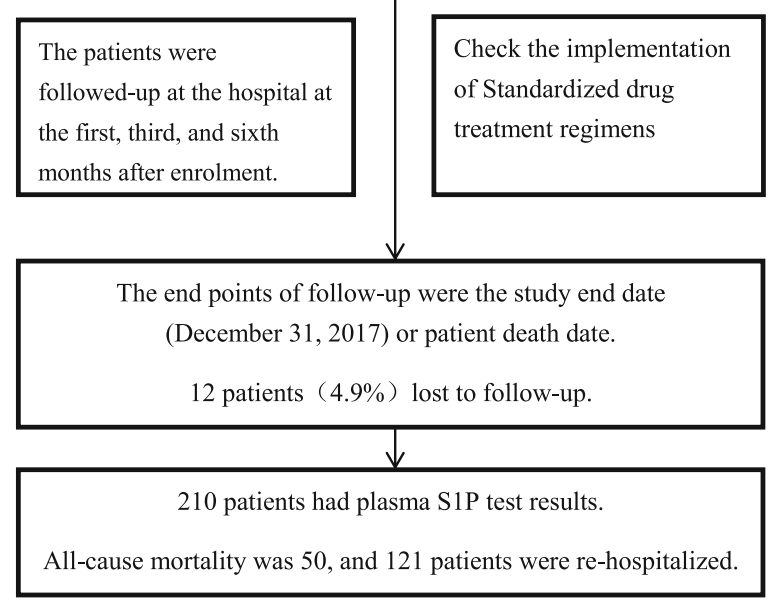

Fig. 1 The study design and population

On the day of enrolment, the patients were examined by uniformly trained physicians and systematically measured for body weight, blood pressure and heart rate. Heart failure symptoms were assessed using the New York Heart Association functional classification, and a 12-lead ECG was performed. Echocardiography was performed using Philips iE33 ultrasound system (Philips, Amsterdam, Netherlands) by experienced cardiologists independent of the 2 hospitals. The left ventricular ejection fraction (LVEF) value was uniformly measured by biplane Simpson rule. At last physicians recorded the treatment regimens.

\section{Blood sample}

Blood samples were obtained by venipuncture and centrifuged at $1700 \mathrm{~g}$ to separate EDTA plasma and serum at once and shipped to a central laboratory with standardized procedures. Serum was collected for analysis including liver, kidney and lipids function-related tests and electrolytes (HITACHI 7180, HITACHI, Tokyo, Japan). Full blood samples were used to test the hematologic parameters (KX 21 $\mathrm{N}$ analyzers, Sysmex, Kobe, Japan). After these tests, all samples were stored at $-80^{\circ} \mathrm{C}$ for future analysis. The serum NT-proBNP levels were detected as a batch analysis in a central laboratory by electrochemiluminescence immunoassay (Roche Diagnostics, Rotkreuz, Switzerland).

Plasma S1P was measured by liquid chromatographytandem mass spectrometry. We used a previously described protocol with minor modifications [14]. Plasma $(100 \mu \mathrm{L})$ was deproteinated by the addition of methanol $(400 \mu \mathrm{L})$. The internal standard D-erythro-C17-sphingosine-1-phosphate $(10 \mu \mathrm{L}, 10 \mu \mathrm{mol} / \mathrm{L}$, Avanti Polar Lipids Inc. Alabaster, USA) with the $\mathrm{m} / \mathrm{z} 366.4$ to 250.4 was used to correct for variations in sample preparation and instrument response. Extracts were cleared by 5810R EPPENDORF centrifugation (12,000 rpm, $10 \mathrm{~min})$ (EPPENDORF, Wesseling-Berzdorf, Germany) and subjected to reverse-phase chromatography on an Agilent Eclipse XDB C-18 analytical column $(2.1 \mathrm{~mm} \times 150 \mathrm{~mm}$, $3.5 \mu \mathrm{m}$ ) (Agilent Technologies Inc. Santa Clara, USA) at 1a flow rate of $0.3 \mathrm{ml} / \mathrm{min}$. S1P was eluted by a ballistic gradient (30 to $85 \%$ methanol, $0.2 \%$ formic acid; $\%=$ volume $\%$ ) and measured by a Shimadzu HPLC system coupled to an API 4000 tandem mass spectrometer (Applied Biosystems/MDS SCIEX, Framingham, USA). The quantification determination was performed using multiple reaction monitoring $(\mathrm{M}+\mathrm{H}$ S1P parent ion). A calibration curve $(0.125-5 \mu \mathrm{mol} / \mathrm{L}$ S1P) was generated to calculate S1P level in samples. The same sample was tested multiple times with a relative standard deviation less than $5 \%$ within $12 \mathrm{~h}$, and less than $10 \%$ within 6 days when stored at room temperature.

\section{Assessment of outcomes}

The primary endpoints were all-cause mortality and hospitalization due to aggravation of heart failure. The occurrence of patient end point events was determined through telephone follow-ups of the patients and their families, a review of hospital medical records and death certificates. All 50 all-cause deaths satisfied the definition of cardiovascular death. Therefore, no further statistical analysis was performed on cardiovascular death as secondary outcome. And we used the "average No. of hospitalization per person per year" to adjust the multiple hospitalizations and different length of follow-up. The follow-up ended on December 31, 2017 or patient death. 


\section{Statistical analysis}

The Kolmogorov-Smirnov test was used to test whether a variable conformed to a normal distribution. Normal distribution variables were expressed as the mean \pm standard deviation (SD), and Student's T test was used for comparisons between groups. Variables that did not conform to a normal distribution were expressed as the median [interquartile 1, interquartile 3], and the MannWhitney $U$ test was used for inter-group comparison. Patients were grouped according to the baseline S1P level quartiles (Quartile1 (Q1), Quartile2 (Q2), Quartile3 (Q3) and Quartile4 (Q4) group). The baseline characteristics among the 4 groups were analyzed by the KruskalWallis test for nonparametric variables, ANOVA for parametric variables, and the $X^{2}$ test for categorical variables. Spearman and Pearson correlation analyses were used to explore the correlations.

We analyzed S1P level as a continuous variable, fitting a restricted cubic spline function with 4 knots (located at the 5th, 35th, 65th, and 95th percentiles), and $1.10 \mu \mathrm{mol} / \mathrm{L}$ (The median S1P level in control group.) was chosen as the reference for all spline plots [15].

Cox proportional hazard analysis was used to determine the relationship between S1P and all-cause death. The Q2 group was selected as the reference category because it has the largely normal S1P levels and associated with the lowest risk of all-cause mortality [16]. We calculated the Meta-analysis Global Group in Chronic Heart Failure (MAGGIC) scores [17] of all patients to fit a multivariable Cox regression model. The multivariable Cox models are shown as follow. Model 1: S1P was adjusted by age and gender. Model 2: S1P was adjusted by MAGGIC score. Model 3: On the basis of Model 2, lgNTproBNP was added. The only missing data in the final model were 2 patients for NT-proBNP $(<1 \%)$, and we replaced the two missing NT-proBNP by the previous nonmissing value. As a result, the final model contained all the 210 observed patients, 50 events and no more than 5 variables which were identified to be independent predictors of all-cause death. The ability of S1P to improve death risk prediction beyond using traditional risk factors was assessed using indexes of discrimination as measured by integrated discrimination improvement (IDI) and reclassification as measured by the net reclassification index (NRI).

Survival curves were using the Kaplan-Meier method and the log-rank test was used for comparison. A 2-tailed $P$ value of less than 0.05 was considered statistically significant. SPSS22.0 (SPSS, Inc., Chicago, IL, USA), STATA13.1 (StataCorp, Inc., Texas, USA) and GraphPad prism 5.0 (GraphPad Software, Inc., Cary, NC) were used for statistical analysis and graphing.

\section{Results}

Baseline and distribution characteristics of S1P levels

Among the CHF patients who completed the follow-up, 210 patients had plasma S1P test results. The patients were grouped according to the baseline plasma S1P level quartiles, and 54 healthy volunteers in the same area were selected as controls. The comparison results of S1P level are shown in Fig. 2. The overall plasma S1P of CHF patients had a higher mean value $(1.269 \mu \mathrm{mol} / \mathrm{L}$ vs $1.122 \mu \mathrm{mol} / \mathrm{L}, P=0.006)$ and a larger standard deviation (0.441 vs $0.316, P=0.022$ ) than those in the control group. Compared to the control group, S1PQ1 was lower, S1PQ3 and S1PQ4 were higher, and S1PQ2 showed no statistical difference.

The baseline data for the entire study population and the occurrence of outcomes in each quartile are presented in Table 1. The average age of the patients was 61.7 (SD 9.3) years, males accounted for $63.8 \%$, LVEF was 35.76 (SD 7.57) \%, NT-proBNP was 1633.5 [857.33$3123.25] \mathrm{pg} / \mathrm{mL}, 9.1 \%$ of patients had a history of diabetes, and $31.3 \%$ had a history of hypertension. Groups with higher S1P levels had a higher red blood cell count $(P<0.001)$, haemoglobin $(P=0.018)$, monocyte count $(P<0.001)$, and percentage of monocytes $(P<0.001)$. The neutrophil count $(P=0.001)$ and percentage of neutrophils $(P=0.007)$ showed the opposite trend. In ultrasonic cardiogram, LVEF\% $(P=0.04)$ and LVEDV $(P=$ $0.039)$ differed between the groups. Although we used the uniform principles, $\beta$-receptor blockers were used more frequently in the $\mathrm{Q} 3$ group, which might be related to the fewer contraindications for drug use. The usage

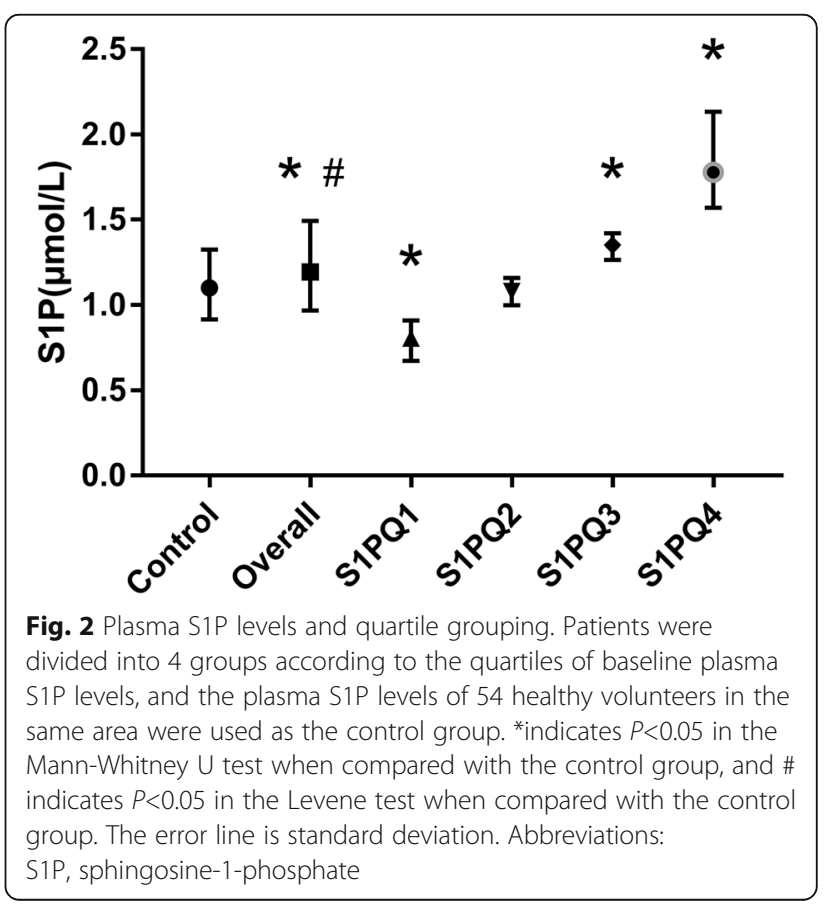




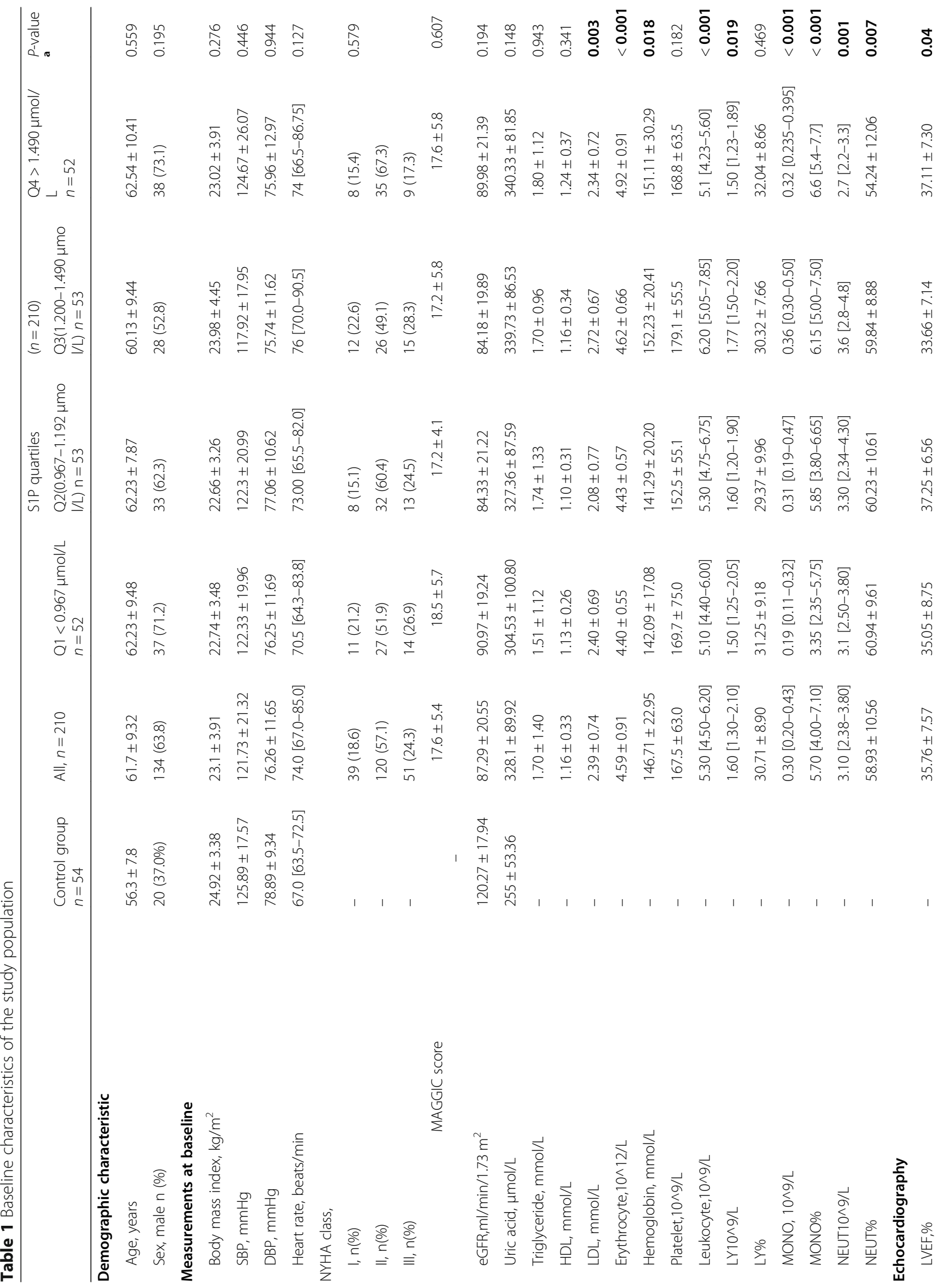




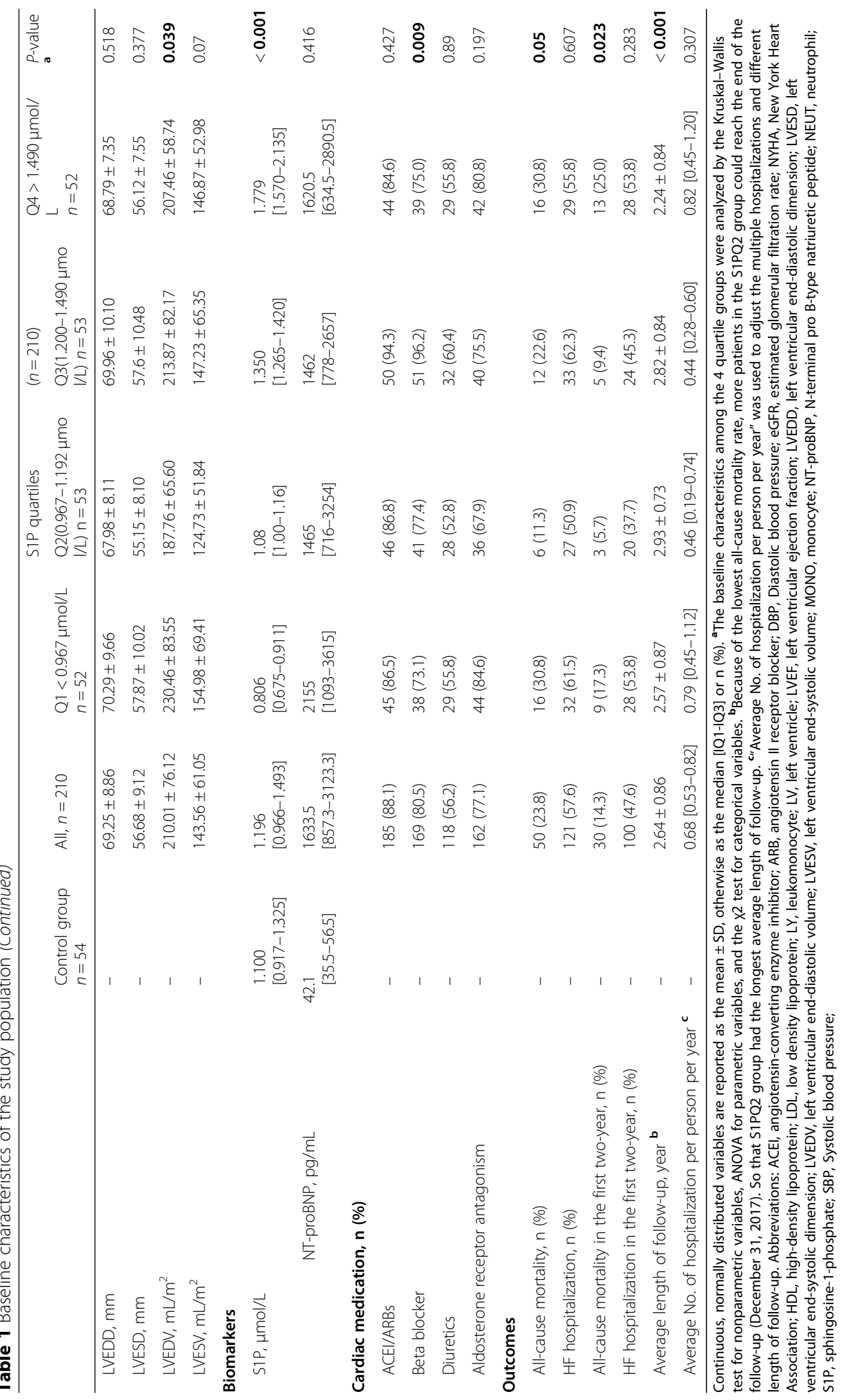


rates of other drugs did not significantly differ between the groups.

The correlation analysis showed that the plasma S1P level was positively correlated with the red blood cell count $(\mathrm{r}=0.312, P<0.001)$, haemoglobin $(\mathrm{r}=0.189, P=$ $0.006)$, monocyte count $(\mathrm{r}=0.287, P<0.001)$ and monocyte percentage $(\mathrm{r}=0.412, P<0.001)$, but it was negatively correlated with the percentage of neutrophils $(\mathrm{r}=$ $-0.263, P<0.001$ ).

\section{Follow-up}

After an average follow-up period of 31.7 (SD 10.3) months (range: 2-43 months), 50 all-cause deaths occurred in the 210 patients, and 121 patients were rehospitalized due to exacerbation of heart failure (Table 1). All 50 all-cause deaths satisfied the definition of cardiovascular death. Therefore, no further statistical analysis was performed on cardiovascular death as secondary outcomes. The Q2 group had the lowest allcause mortality and proportion $(n=6,11.3 \%)$, while Q3 $(n=12,22.6 \%), \mathrm{Q} 4(n=16,30.8 \%)$ and Q1 group $(n=$ $16,30.8 \%$ ) showed increased all-cause mortality. The similar trend could be found in all-cause mortality and hospitalization rates during the first 2 years of follow-up and the average No. of hospitalizations per person per year. However, there was no significant difference in the HF re-hospitalization rate between the groups.

\section{Prognostic value of baseline S1P levels}

Based on univariable Cox regression with restricted cubic spline analysis, a non-linear and U-shaped association between S1P levels and risk of all-cause death was observed (Fig. S1). And the trend still existed in adjusted HR spline (Fig. 3). The inflection point of U-shaped spline was $1.06 \mu \mathrm{mol} / \mathrm{L}$. Patients with a S1P level of $1.06 \mu \mathrm{mol} / \mathrm{L}$ had the lowest risk of all-cause death, which was consistent with the results in Fig. 2, where the S1P level of the Q2 group and the control group did not significantly differ. We divided patients into two groups according to the inflection point. In the group with S1P level $\geq 1.06 \mu \mathrm{mol} / \mathrm{L}$, patients with lower S1P had significantly better survival (Adjusted $\mathrm{HR}=2.368$ [1.0065.572], $P=0.048$ ). In patients with S1P level $<1.06 \mu \mathrm{mol} /$ $\mathrm{L}$, a greater decrease in S1P level was associated with increased risk of all-cause death (Adjusted $\mathrm{HR}=$ 0.041[0.002-0.808], $P=0.036$ ). The U-shaped association was robust in the multivariate analyses (Table S1).

Then we analyzed S1P level as a category variable according to the quartiles. The Cox proportional hazard analysis results are shown in Table 2. The patient plasma S1P level belonging to the Q1 group, the patient plasma S1P level belonging to the Q4 group, lgNT-proBNP, DBP, uric acid, haemoglobin, LVEF, LVEDD, LVESD, LVEDV and LVESV were found to be univariate

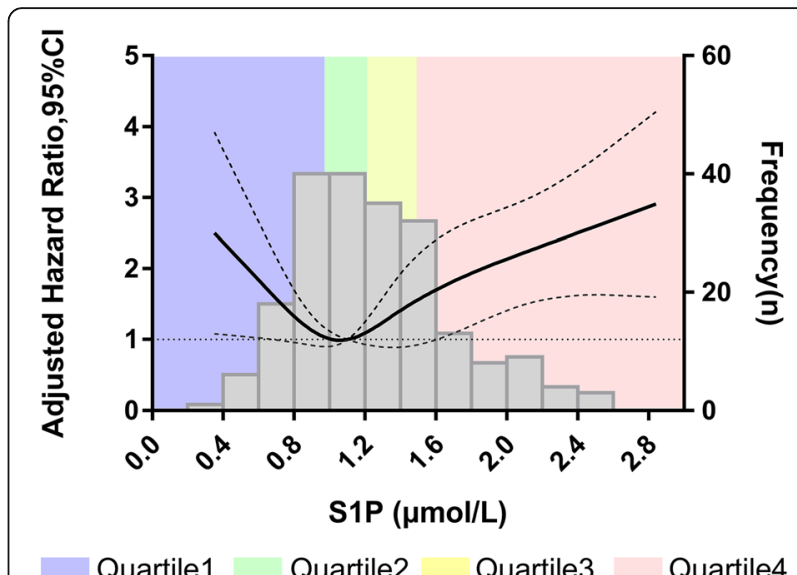

Fig. 3 Association between S1P levels and the adjusted hazard ratio for all-cause death. Histograms stand for frequency of patients at different S1P concentrations (The right-hand $y$-axis). The solid curve gives expected of adjusted HR based on restricted cubic spline analysis (The left-hand y-axis). The dashed curves represent the 95\% confidence intervals for expected of adjusted HR (The left-hand $y$ axis). Model adjusted on MAGGIC score and IgNT-proBNP

predictors of all-cause death. Univariable analysis revealed that low S1P levels (Q1 group: S1P $<0.967 \mu \mathrm{mol} /$ L; HR: $3.271(1.277-8.381), P=0.014)$ and high S1P levels (Q4 group: S1P > 1.490 $\mu \mathrm{mol} / \mathrm{L}$; HR: 3.870 (1.5049.960), $P=0.005$ ) were both associated with the high risk of all-cause death. Multivariable analysis showed that patient plasma S1P level belonging to the $\mathrm{Q} 4$ group (adjusted HR 3.418; 95\%CI 1.320-8.849; $P=0.011$ ) were independent predictors of all-cause mortality, while the prognostic value of patient plasma S1P level belonging to the Q1 group (adjusted HR: 2.525; 95\%CI: $0.972-$ 6.563; $P=0.057$ ) subsided after additional adjustment for MAGGIC score and lgNT-proBNP.

According to the S1P optimal thresholds obtained by the restricted cubic spline, patients can be divided into high risk group and low risk group. Adding S1P classification to the model with MAGGIC score and lgNTproBNP, we found a $39.8 \%(\mathrm{Z}=2.425, P=0.015) \mathrm{im}$ provement in the NRI (Table S2) and a $0.027(\mathrm{Z}=2.226$, $P=0.026)$ improvement in the IDI.

The survival curves in Fig. 4 showed that plasma S1P levels in the lowest quartile $(\mathrm{S} 1 \mathrm{P}<0.967 \mu \mathrm{mol} / \mathrm{L})$ and highest quartile $(\mathrm{S} 1 \mathrm{P}>1.490 \mu \mathrm{mol} / \mathrm{L})$ were at a higher risk of death. But there was no significant difference in the re-hospitalization rate due to aggravated heart failure between the groups.

\section{Discussion}

The main finding of this study was that plasma S1P in patients with heart failure was associated with all-cause mortality, which showed a U-shaped correlation. And we also described the overall distribution of S1P levels in 
Table 2 Cox regression analysis for S1P and all-cause death

\begin{tabular}{|c|c|c|c|c|}
\hline Model & HR ratio $(95 \% \mathrm{Cl})$ for S1PQ1 & $P$-value & HR ratio(95\%Cl) for S1PQ4 & $P$-value \\
\hline S1P Crude HR & $3.271(1.277-8.381)$ & 0.014 & $3.870(1.504-9.960)$ & 0.005 \\
\hline Model 1 S1P + age + gender & $3.267(1.274-8.378)$ & 0.014 & 3.885 (1.508-10.008) & 0.005 \\
\hline \multicolumn{5}{|c|}{ Adjustments for predictors of all-cause death in CHF } \\
\hline S1P + IgNT-proBNP & $2.634(1.019-6.808)$ & 0.046 & $3.504(1.362-9.017)$ & 0.009 \\
\hline $\mathrm{S} 1 \mathrm{P}+\mathrm{DBP}$ & $3.197(1.248-8.188)$ & 0.015 & $3.984(1.546-10.265)$ & 0.004 \\
\hline S1P + LVEF\% & $2.785(1.072-7.235)$ & 0.036 & $3.742(1.453-9.637)$ & 0.006 \\
\hline S1P + LVEDD & $2.922(1.131-7.546)$ & 0.027 & $3.723(1.447-9.578)$ & 0.006 \\
\hline S1P + LVESD & $2.850(1.101-7.378)$ & 0.031 & $3.701(1.438-9.526)$ & 0.007 \\
\hline S1P + LVEDV & $2.689(1.032-7.007)$ & 0.043 & $3.504(1.360-9.030)$ & 0.009 \\
\hline S1P + LVESV & $2.759(1.063-7.164)$ & 0.037 & $3.415(1.321-8.825)$ & 0.011 \\
\hline \multicolumn{5}{|c|}{ Adjustments for independent predictors of all-cause death in $\mathrm{CHF}$} \\
\hline S1P + IgNT-proBNP+LVEDV & $2.043(0.771-5.413)$ & 0.115 & $3.144(1.220-8.101)$ & 0.018 \\
\hline S1P + IgNT-proBNP+DBP & $2.420(0.932-6.281)$ & 0.069 & $3.342(1.291-8.655)$ & 0.013 \\
\hline S1P + LVEDV+DBP & $2.574(0.981-6.750)$ & 0.055 & $3.736(1.449-9.630)$ & 0.006 \\
\hline Model 2 S1P + MAGGIC score & $2.838(1.097-7.344)$ & 0.032 & $3.878(1.505-9.991)$ & 0.005 \\
\hline Model 3 S1P + MAGGIC score + IgNT-proBNP & $2.525(0.972-6.563)$ & 0.057 & $3.418(1.320-8.849)$ & 0.011 \\
\hline
\end{tabular}

Abbreviations: HR hazard ratio; MAGGIC score Meta-analysis Global Group in Chronic Heart Failure score; NT-proBNP N-terminal pro B-type natriuretic peptide; S1P sphingosine-1-phosphate

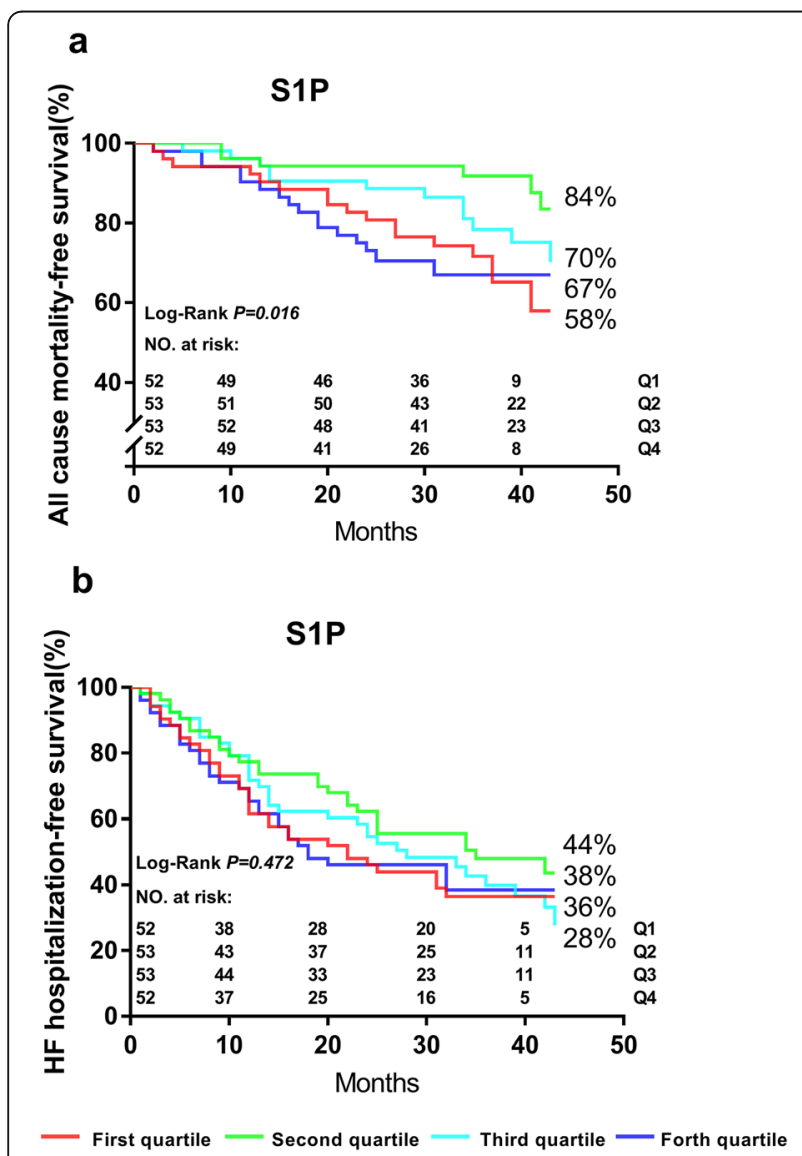

Fig. 4 Kaplan-Meir curves of all-cause mortality (a) and heart failure hospitalization (b). Abbreviations: S1P, sphingosine-1-phosphate heart failure patients and first provided CHF patients normal (low risk) S1P thresholds for reference.

\section{U-shaped relationship bridges the gap from basic study to clinic patients}

In this study, we found that S1P had different distribution characteristics in the normal population and CHF patients. Abnormally increased (Q3, Q4 group), decreased (Q1 group) and roughly normal S1P levels (Q2 group) coexisted in CHF patients. Because abnormal decrease had less amplitude and room than increase, the CHF group showed a higher mean value $(1.269 \mu \mathrm{mol} / \mathrm{L}$ vs $1.122 \mu \mathrm{mol} / \mathrm{L}, \quad P=0.006)$ and standard deviation (0.441 vs $0.316, P=0.022)$ of S1P level than the control group. Combined with the restricted cubic spline results, where patients with roughly normal S1P levels (Q2 group) had the lowest mortality rate, these results suggest that the S1P level is associated with all-cause mortality in a U-shaped relationship, indicating that there may be a normal value range for S1P levels in the middle of the entire distribution rather than on either side.

In previous animal model studies, different results were reported for the S1P/S1PR axis function.

Some of the studies have found that S1P and S1PR expression decreased after heart injury which can be improved by upregulating S1P level or S1PR activity. In the model of post-ischemic HF, Cannavo A et al. demonstrated that either cardiac or circulating levels of S1P are reduced compared to non-ischemic control [6]. S1PR1 receptor expression decreased after myocardial 
infarction, and chronic S1P treatment could arrest postMI HF progression [5]. Meanwhile, S1P lyase activation in the myocardium following ischemia leads to reduced S1P levels and that knockout mice for this enzyme exhibit higher S1P levels and smaller infarct size [18]. In addition to the ischemia model, Yan $\mathrm{H}$ et al. [19] demonstrated that exogenous S1P improved rat cardiac hypertrophy induced in the transverse aortic constriction model. Similar conclusions were reached in studies of FTY720 (A kind of high-affinity agonist of S1PR1 and S1PR3-5). Hofmann U et al. [20] have tested the hypothesis that FTY720 can improve functional recovery when applied with reperfusion after myocardial ischaemia. Yeh CC et al. [10] showed that S1P levels decreased after myocardial infarction in rats and that administration of FTY720 reduced apoptosis and ventricular remodelling after infarction. Santos-Gallego CG et al. [21] found that S1PR activation with FTY720 during acute MI reduced infarct size via the reperfusion injury salvage kinase and survivor activating factor enhancement pathways, improved systolic LV function, and mitigated postMI LV remodeling.

Other studies have found that S1P levels increased after heart injury. Zhang F et al. [22] showed that plasma S1P levels, Sphk1 and S1PR1 activities increased after myocardial infarction in rats and that downregulation of S1P function by an inhibitor could protect the heart. In another mouse model study [23], 10-week-old C57Bl6 mice underwent experimental myocardial infarction. At 1 to 12 weeks postoperative, S1P was increased as early as 1 week after MI and remained elevated. Empinado $\mathrm{HM}$ et al. [24] made similar findings. In their research myocardial infarction was used to induce $\mathrm{CHF}$ in rats. Heart failure increased diaphragm S1P level. They found that the levels of S1P in CHF group were increased twofold than control group.

These studies used different animal models, observed different time points during the disease process, and identified different S1P change directions. But fortunately, the similarity of these studies is that either an increase or a decrease of S1P levels in heart failure animal models is harmful, and regulation of abnormal S1P level towards the control level is beneficial for heart protection. We identified both elevated and decreased S1P levels in clinical patients and a U-shaped association with all-cause deaths, allowing different directions of previous animal model studies to be simultaneously reproduced at the clinical level.

In summary, before this study, there was insufficient information to suggest that either upregulate or downregulate S1P signaling will be effective in the setting of heart failure [11]. For the first time, our study showed a U-shaped relationship between the S1P levels and allcause death, and provided CHF patients normal (low risk) S1P thresholds for reference. Through restoring abnormal levels to a normal range instead of simply upregulation or down-regulation, S1P has the potential to be a therapeutic target for reducing the risk of death in patients with heart failure in the future.

\section{U-shaped relationship bridges the gap between different mechanisms}

At present, excessive activation of the neurohumoral endocrine system (Sympathetic nervous system and Renin-angiotensin system) and its consequent progressive ventricular remodeling are the most recognized mechanisms leading to the development of heart failure. Although an increasing body of experimental evidence supports the notion that normal S1P/S1PR system protects the heart, there is insufficient evidence to support that abnormal S1P is directly involved in the development of heart failure. Our study has the inherent limitation of observational research, that is, the causal relationship between abnormal S1P level and the development of heart failure cannot be explained from the mechanism, but it can provide hypothesis for the direction of future research.

In this study, the prognostic value of decreased plasma S1P levels for all-cause mortality diminished after correction with NT-proBNP and LVEDV. The LVEDV is an independent risk factor for all-cause death and also presents the degree of left ventricular remodeling in heart failure. The S1PQ1 group had higher LVEDV than S1PQ2 group (230.46 (SD 83.55) vs 187.76 (SD 65.60), $P=0.007$ ). And Cannavo A et al. [5] showed a mutual regulation mechanism between S1P/S1PR function and $\beta$-adrenergic receptor function. These results suggest that an abnormal decline of plasma S1P may be associated with heavier ventricular remodelling. Furthermore, Gazit SL et al. [25] showed that S1P deficiency may aggravate vasoplegia, arguing a vital role for S1P in maintaining vascular resistance during recovery from circulatory shock. The results may explain the relationship between the increased all-cause mortality and low S1P levels in some patients.

The prognostic value of an abnormal elevation of sphingosine-1-phosphate (S1P) for death is completely independent of the previous high-risk factors and the prediction indexes associated with heart failure. After adjusting for variables associated with S1P and risk factors for all-cause death in heart failure, the prognostic value of S1P was not reduced, suggesting the presence of a new mechanism for the occurrence and development of heart failure that is independent of previously identified conventional risk factors. As an endogenous sphingolipid molecule with important biological activity, S1P has important immunomodulatory functions [26, 27] that S1P/S1PR abnormally changes in autoimmune 
diseases such as rheumatoid arthritis [28] and in severe infectious diseases such as sepsis [14]. The excessive activation of S1P/S1PR can also lead to the occurrence of immune disorders and inflammation [29], which is also one mechanism of heart failure. The cause of S1P changes in heart failure patients, the mechanism by which excessive activation of the S1P/S1PR axis leads to increased all-cause mortality in these patients, and the relationship between the S1P/S1PR axis and immune disorders await further research for confirmation.

We hypothesize that during the occurrence and development of heart failure, the two different directions of abnormal changes in plasma S1P levels are linked to the two different mechanisms of ventricular remodelling and immune disorders, separately or simultaneously. S1P may have the potential to act as the "bridge molecule" that links the neurohumoral endocrine system and the immune system, which can be investigated in future studies on the pathophysiological process of heart failure occurrence and development.

\section{Limitations}

There are several limitations related to our study. Because out-of-hospital CHF patients were enrolled in this study, some patients did not undergo coronary angiography, so in these cases the patients were not classified according to the aetiology of heart failure. As a result, whether different causes could affect the direction of plasma S1P could not be analysed in depth. Furthermore, we do not know the variance of $\mathrm{S} 1 \mathrm{P}$ value if it is measured at different time points in the same patient at present. Although the all-cause mortality during the whole follow-up period and in the first two-year showed the same trend, we still wonder whether it is more appropriate to use the sequential value of S1P to predict the prognosis more than 2 years after the enrolment. Moreover, only patients with heart failure in 2 hospitals were enrolled in this study. Due to these limitations, we consider our study mainly as a hypothesis generator. Future large-scale, multicenter clinical research will effectively improve the reliability of these findings.

\section{Conclusions}

In patients with chronic systolic heart failure, plasma S1P levels are related to the long-term all-cause mortality with a U-shaped correlation. Through restoring abnormal levels to a normal range instead of simply up regulation or down regulation, S1P may have the potential to be a therapeutic target for reducing the risk of death in patients with heart failure in the future.

\section{Supplementary information}

Supplementary information accompanies this paper at https://doi.org/10. 1186/s12944-020-01262-2.

Additional file 1. Table S1. Univariate and multivariate Cox analyses for S1P in two subgroups. Table S2. Net reclassification of death and not death with adding S1P to Risk Factors. Figure S1. Association between S1P levels and the hazard ratio for all-cause death.

\section{Abbreviations}

AV block: Atrioventricular block; ACEl: Angiotensin-converting enzyme inhibitor; ARB: Angiotensin II receptor blocker; CHF: Chronic heart failure; DBP: Diastolic blood pressure; ECG: Electrocardiograph; EDTA: Ethylene diamine tetraacetic acid; EF: Ejection fraction; eGFR: Estimated glomerular filtration rate; HDL: High-density lipoprotein; HR: Hazard ratio; LDL: Low density lipoprotein; LV: Left ventricle; LVEF: Left ventricular ejection fraction; LVEDD: Left ventricular end-diastolic dimension; LVESD: Left ventricular endsystolic dimension; LVEDV: Left ventricular end-diastolic volume; LVESV: Left ventricular end-systolic volume; LY: Leukomonocyte; MONO: Monocyte; NEUT: Neutrophil; NT-proBNP: N-terminal pro B-type natriuretic peptide; NYHA: New York Heart Association; S1P: Sphingosine-1-phosphate; S1PR: Sphingosine-1-phosphate receptor; SBP: Systolic blood pressure; SD: Standard deviation

\section{Acknowledgements}

Not applicable.

\section{Conflict of interest}

None.

\section{Support grants}

This work was supported by the Ministry of Science and Technology and the Ministry of Finance of the People's Republic of China. [Grant numbers: No.2012GS610101 and No. 2017YFC1308302]

\section{Authors' contributions}

$X Y$ conducted experiments, performed statistical evaluations, and wrote the manuscript. JW provided methodology and formal analysis. JP provided methodology. LQ performed statistical evaluations. MQ, WX, WY, ZJ, LJ, LY, $L, W L, D C, L H, X Z$ obtained samples from patients, recorded patient history, assessed the clinical characteristics of patients and performed interviews during the enrolment process. CS, SB supervised sample collection. BL provided feedback on the manuscript and funding acquisition. MA conceived the idea, designed the study, obtained necessary permissions, provided scientific support and funding acquisition. The author(s) read and approved the final manuscript.

\section{Funding}

This work was supported by the Ministry of Science and Technology and the Ministry of Finance of the People's Republic of China. [Grant numbers: No.2012GS610101 and No. 2017YFC1308302].

\section{Availability of data and materials}

The datasets generated during and/or analysed during the current study are available from the corresponding author on reasonable request.

\section{Ethics approval and consent to participate}

Ethical approval was obtained from the ethics committee of the First Affiliated hospital of Xi'an Jiaotong University (2013-133). All patients and controls provided written informed consent. All steps of the study conformed to the Helsinki Declaration and Good Clinical Practice guidelines.

\section{Consent for publication}

Not applicable.

Competing interests

The authors declare that they have no competing interests. 


\section{Author details}

'Department of Cardiovascular Medicine, First Affiliated Hospital of Xi'an Jiaotong University; Shaanxi Key Laboratory of Molecular Cardiology; Key Laboratory of Environment and Genes Related to Diseases, Ministry of Education, No. 277 West Yanta Road, Xi'an 710061, Shaanxi Province, People's Republic of China. ${ }^{2}$ Department of Pharmacy, the Second Affiliated Hospital of Air Force Medical University, No. 1 Xinsi Road, Xi'an, Shaanxi, China. ${ }^{3}$ Key Laboratory of Resource Biology and Biotechnology in Western China, College of Life Sciences, Northwest University, No. 299 Taibai Road, Xi'an, Shaanxi, China. ${ }^{4}$ Department of Epidemiology and Biostatistic, School of Public Health Xi'an Jiaotong University Health Science Center, No. 76 West Yanta Road, Xi'an, Shaanxi, China. ${ }^{5}$ The Affiliated Hospital of Qingdao University, No. 1 Jiangsu Road, Qingdao, Shandong, China. ${ }^{6}$ Department of Cardiology, the First Affiliated Hospital, Sun Yat-Sen University, No. 58 Zhongshan Road, Guangzhou, China

Received: 20 December 2019 Accepted: 8 April 2020

Published online: 04 June 2020

\section{References}

1. Ponikowski P, Voors AA, Anker SD, et al. 2016 esc guidelines for the diagnosis and treatment of acute and chronic heart failure: the task force for the diagnosis and treatment of acute and chronic heart failure of the european society of cardiology (esc). Developed with the special contribution of the heart failure association (hfa) of the esc. Eur Heart J. 2016;37(27):2129-200.

2. Ej B, Muntner P, Alonso A, et al. Heart disease and stroke statistics-2019 update: a report from the american heart association. Circulation. 2019; 139(10):e56-e528.

3. Spiegel S, Milstien S. The outs and the ins of sphingosine-1-phosphate in immunity. Nat Rev Immunol. 2011;11(6):403-15.

4. Takabe K, Paugh SW, Milstien S, Spiegel S. "Inside-out" signaling of sphingosine-1-phosphate: therapeutic targets. Pharmacol Rev. 2008;60(2): $181-95$.

5. Cannavo A, Rengo G, Liccardo D, et al. Beta1-blockade prevents postischemic myocardial decompensation via beta3ar-dependent protective sphingosine-1 phosphate signaling. J Am Coll Cardiol. 2017;70(2):182-92.

6. Cannavo A, Rengo G, Liccardo D, et al. Beta1-adrenergic receptor and sphingosine-1-phosphate receptor 1 (s1 pr1) reciprocal downregulation influences cardiac hypertrophic response and progression to heart failure: protective role of s1pr1 cardiac gene therapy. Circulation. 2013;128(15): 1612-22.

7. Theilmeier G, Schmidt C, Herrmann J, et al. High-density lipoproteins and their constituent, sphingosine-1-phosphate, directly protect the heart against ischemia/reperfusion injury in vivo via the s1p3 lysophospholipid receptor. Circulation. 2006;114(13):1403-9.

8. Otaka N, Shibata R, Ohashi K, et al. Myonectin is an exercise-induced myokine that protects the heart from ischemia-reperfusion injury. Circ Res. 2018;123(12):1326-38.

9. Takuwa N, Ohkura S, Takashima S, et al. S1p3-mediated cardiac fibrosis in sphingosine kinase 1 transgenic mice involves reactive oxygen species. Cardiovasc Res. 2010;85(3):484-93.

10. Yeh CC, Li H, Malhotra D, Huang MC, et al. Sphingolipid signaling and treatment during remodeling of the uninfarcted ventricular wall after myocardial infarction. Am J Physiol Heart Circ Physiol. 2009;296(4):H1193-9.

11. Mann DL. Sphingosine 1-phosphate as a therapeutic target in heart failure: more questions than answers. Circulation. 2012;125(22):2692-4.

12. Karliner JS. Sphingosine kinase and sphingosine 1-phosphate in the heart: a decade of progress. Biochim Biophys Acta. 2013;1831(1):203-12.

13. McMurray JJ, Adamopoulos S, Anker SD, et al. ESC guidelines for the diagnosis and treatment of acute and chronic heart failure 2012: the task force for the diagnosis and treatment of acute and chronic heart failure 2012 of the European Society of Cardiology. Developed in collaboration withthe heart failure association (HFA) of the ESC. Eur Heart J. 2012;33(14):1787-847.

14. Winkler MS, Nierhaus A, Holzmann M, et al. Decreased serum concentrations of sphingosine-1-phosphate in sepsis. Crit Care. 2015;19:372. https://doi.org/10.1186/s13054-015-1089-0.

15. Harrell F. Regression Modelling strategies with applications to linear models, LogisticRegression, and survival analysis. New York, NY: SpringerVerlag; 2001.
16. O'Donnell MJ, Yusuf S, Mente A, et al. Urinary sodium and potassium excretion and risk of cardiovascular event. JAMA. 2011:306(20):2229-38.

17. Pocock SJ, Ariti CA, McMurray JJ, et al. Predicting survival in heart failure: a risk score based on 39372 patients from 30 studies. Eur Heart J. 2013;34(19): 1404-13.

18. Bandhuvula P, Honbo N, Wang GY, et al. S1P lyase: a novel therapeutic target for ischemia-reperfusion injury of the heart. Am J Physiol Heart Circ Physiol. 2011;300(5):H1753-61. https://doi.org/10.1152/ajpheart.00946.2010 Epub 2011 Feb 18

19. Yan H, Yi S, Zhuang H, Wu L, Wang DW, Jiang J. Sphingosine-1-phosphate ameliorates the cardiac hypertrophic response through inhibiting the activity of histone deacetylase-2. Int J Mol Med. 2018;41(3):1704-14.

20. Hofmann U, Burkard N, Vogt C, et al. Protective effects of sphingosine-1phosphate receptor agonist treatment after myocardial ischaemiareperfusion. Cardiovasc Res. 2009:83(2):285-93.

21. Santos-Gallego CG, Vahl TP, Goliasch G, et al. Sphingosine-1-phosphate receptor agonist fingolimod increases myocardial salvage and decreases adverse postinfarction left ventricular remodeling in a porcine model of ischemia/reperfusion. Circulation. 2016;133(10):954-66.

22. Zhang F, Xia Y, Yan W, et al. Sphingosine 1-phosphate signaling contributes to cardiac inflammation, dysfunction, and remodeling following myocardial infarction. Am J Physiol Heart Circ Physiol. 2016;310(2):H250-61.

23. Hoefer J, Azam MA, Kroetsch JT, et al. Sphingosine-1-phosphate-dependent activation of p38 MAPK maintains elevated peripheral resistance in heart failure through increased myogenic vasoconstriction. Circ Res. 2010;107(7): 923-33.

24. Empinado HM, Deevska GM, Nikolova-Karakashian M, et al. Diaphragm dysfunction in heart failure is accompanied by increases in neutral sphingomyelinase activity and ceramide content. Eur J Heart Fail. 2014;16(5): 519-25.

25. Gazit SL, Mariko B, Thérond P, et al. Platelet and erythrocyte sources of S1P are redundant for vascular development and homeostasis, but both rendered essential after plasma S1P depletion in anaphylactic shock. Circ Res. 2016;119(8):e110-26.

26. Yanagida K, Hla T. Vascular and immunobiology of the circulatory sphingosine 1-phosphate gradient. Annu Rev Physiol. 2017;79:67-91.

27. Rosen H, Stevens RC, Hanson M, Roberts E, Oldstone MB. Sphingosine-1phosphate and its receptors: structure, signaling, and influence. Annu Rev Biochem. 2013;82:637-62.

28. Choi HS, KHA-Ohoo K, Jin S, et al. Decreased expression of sphingosine-1phosphate receptor 1 in the blood leukocyte of rheumatoid arthritis patients. Immune Netw. 2018;18(5):e39. https://doi.org/10.4110/in.2018.18.e39.

29. Liang J, Nagahashi M, Ey K, et al. Sphingosine-1-phosphate links persistent stat3 activation, chronic intestinal inflammation, and development of colitisassociated cancer. Cancer Cell. 2013;23(1):107-20.

\section{Publisher's Note}

Springer Nature remains neutral with regard to jurisdictional claims in published maps and institutional affiliations.

Ready to submit your research? Choose BMC and benefit from:

- fast, convenient online submission

- thorough peer review by experienced researchers in your field

- rapid publication on acceptance

- support for research data, including large and complex data types

- gold Open Access which fosters wider collaboration and increased citations

- maximum visibility for your research: over $100 \mathrm{M}$ website views per year

At BMC, research is always in progress.

Learn more biomedcentral.com/submissions 